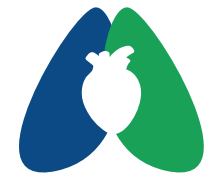

ASSOBRAFIR C I Ê N C I A

\title{
Função pulmonar em pré-escolares e escolares com histórico de bronquiolite viral aguda: revisão da literatura
}

\author{
Pulmonary function in preschoolers and schoolchildren with a \\ history of acute viral bronchiolitis: a systematic review
}

Débora Garcia Oliveira1 (1); Vivian Mara Gonçalves de Oliveira Azevedo²* (1)

\begin{abstract}
Resumo
Introdução: As doenças respiratórias agudas permanecem como importante causa de morbimortalidade, principalmente em crianças, sendo a bronquiolite viral aguda (BVA) a de maior relevância clínica. Objetivo: Realizar uma revisão da literatura sobre a função pulmonar de crianças na idade pré-escolar e escolar com histórico de hospitalização por bronquiolite viral aguda. Métodos: Revisão bibliográfica realizada utilizando as bases de dados on-line PubMed e Biblioteca Virtual em Saúde (BVS), tendo como descritores: "bronchiolitis viral", "spirometry", "respiratory function test" e "child". Foram selecionados artigos em inglês, português e espanhol, publicados entre os anos de 2010 e 2019, que possuíam no título, resumo ou corpo do texto relação com o objetivo da pesquisa. Resultados: Foram incluídos cinco artigos nesta revisão. O tamanho da amostra nos estudos selecionados variou de 25 a 117 pacientes com BVA e a idade dos individuos avaliados variou de 5 a 13 anos - momento em que já eram capazes de realizar o teste espirométrico adequadamente. Os achados da função pulmonar dessas crianças se mostrou reduzido em alguns estudos, o que levou a uma piora da função e quadros de asma, enquanto outras investigações não observaram alterações significativas. Essa redução da função pode ser um marcador da persistência de doenças respiratórias da infância à vida adulta. Conclusão: Os achados da revisão evidenciaram que a BVA no primeiro ano de vida foi fator de risco para sibilância, asma e pior função pulmonar em crianças dos 6 aos 13 anos de idade.
\end{abstract}

Palavras-chave: Bronquiolite viral; Espirometria; Testes de função respiratória; Criança.

\begin{abstract}
Background: Acute respiratory diseases remain an important cause of morbidity and mortality, especially in children, with acute viral bronchiolitis (AVB) being the most clinically relevant. Aim: To conduct a review of the literature about the children's pulmonary function of preschool and school age with a history of hospitalization for acute viral bronchiolitis. Methods: Literature review carried out using the online databases PubMed and Virtual Health Library (VHL), having as descriptors: "viral bronchiolitis", "spirometry", "respiratory function test" and "child". Articles in English, Portuguese and Spanish, published between 2010 and 2019, which had a relationship with the research objective in the title, abstract or body of the text were selected. Results: Five articles were included in this review. The sample size in the selected studies ranges from 25 to 117 patients with AVB and an age of individuals subject to a variation of 5 to 13 years - a time when they were already able to perform or test spirometry used. The findings of the children's pulmonary function were shown to be reduced in few studies, which led to a worsening of function and asthma, while other studies did not observe significant changes. This reduction in function may be a marker of the persistence of respiratory diseases from childhood to adulthood. Conclusion: The review findings showed that AVB in the first year of life was a risk factor for wheezing, asthma and worse lung function in children aged 6 to 13 years.
\end{abstract}

Keywords: Bronchiolitis, viral; Spirometry; Respiratory function tests; Child.

\section{INTRODUÇÃO}

Nos países em desenvolvimento, as doenças respiratórias agudas permanecem como importante causa de morbimortalidade, principalmente em crianças ${ }^{1}$ Dentre estas doenças que acometem lactentes, a bronquiolite viral aguda (BVA) é

*Autor correspondente: Vivian Mara Gonçalves de Oliveira Azevedo E-mail: viviangazevedo1@gmail.com

Este é um artigo publicado em acesso aberto (Open Access) e distribuído sob a licença Creative Commons Attribution NonComercial ShareAlike License, que permite uso, distribuição e reprodução em qualquer meio, sem restrições desde que sem fins comerciais e que o trabalho original seja corretamente citado e de forma que não indique endosso ao trabalho feito. Adicionalmente, qualquer trabalho derivado deverá ser publicado sob a mesma licença. 
a de maior relevância clínica, devido a morbidade elevada, particularmente nos meses de outono e inverno, época sazonal e de circulação do vírus sincicial respiratório (VSR), seu principal agente etiológico² Apesar de sabermos que outros vírus, tais como rinovírus, influenza A e B, parainfluenza, metapneumovírus, adenovírus, papilomavírus e bocavírus (HBoV), também estão envolvidos com a BVA ${ }^{3}$ seus reais efeitos precisam ser melhor estudados.

As crianças acometidas por BVA apresentam, inicialmente, sintomas do trato respiratório alto como rinorreia, tosse, seguido de acometimento do trato respiratório inferior com inflamação, edema e necrose de células epiteliais de pequenas vias aéreas com aumento na produção de muco e broncoespasmo, cujo diagnóstico é eminentemente clínico ${ }^{4}$ É, portanto, considerada a principal causa de hospitalização nos primeiros anos de vida ${ }^{5}$

Dados de estudos epidemiológicos demonstram que, após uma BVA com hospitalização, cerca de 50\% das crianças podem apresentar pelo menos uma ocorrência de sibilância antes do primeiro ano de vida, com reincidência de crises de sibilância ou asma de $30-40 \%$ nos primeiros anos de vida, o que causa morbidade respiratória a longo prazo com diminuição da função pulmonar ${ }^{6,7}$

Há também evidências de que muitas crianças que não possuem sintomas aparentes após BVA, apresentem função pulmonar reduzida e aumento da reatividade das vias aéreas na idade escolar (4-6 anos), o que pode persistir até a idade adulta ${ }^{6,8}$ Esses efeitos após um longo período podem reduzir a qualidade de vida e aumentar a utilização de recursos de saúde para doenças respiratórias tais como: internações hospitalares, atendimentos ambulatoriais e uso de medicamentos ${ }^{9,10}$

Para avaliação da função pulmonar, a espirometria é o teste mais comumente utilizado que permite a quantificação dos distúrbios ventilatórios, mas que exige a compreensão e colaboração do paciente, equipamentos adequados, além de técnicas padronizadas aplicadas por profissionais treinados ${ }^{11} \mathrm{O}$ exame convencional possibilita avaliar todos os volumes, capacidades e fluxos diretamente, com exceção do volume residual (VR), fornecendo ricas informações sobre a função pulmonar ${ }^{12}$

Até o presente momento, foram localizados poucos estudos que investigaram a função pulmonar de crianças em idade pré-escolar e escolar com histórico de hospitalização por BVA nos primeiros anos de vida. Assim, o objetivo deste estudo foi realizar uma revisão na literatura, identificando a associação entre hospitalização por BVA na primeira infância e as consequências respiratórias como piora na função pulmonar e sibilância na idade escolar. Essas morbidades respiratórias de longo prazo impactam no estado de saúde das crianças podendo reduzir a qualidade de vida, sendo necessário maior elucidação.

\section{MÉTODOS}

Uma revisão da literatura foi realizada, e para a seleção dos estudos foram utilizados os seguintes descritores: "bronchiolitis viral", "spirometry", "respiratory function test" e "child". Esses foram selecionados de acordo com as listas de descritores DeCS e MeSH. Para a pesquisa, foram utilizadas as bases de dados on-line PubMed e Biblioteca Virtual em Saúde (BVS), que engloba LILACS, Índice Bibliográfico Español en Ciencias de la Salud, MEDLINE, Biblioteca Cochrane e SciELO. A busca foi realizada no período entre novembro de 2019 e janeiro de 2020, a partir do cruzamento dos descritores eleitos.

Foram selecionados artigos em inglês, português e espanhol, publicados entre os anos de 2010 e 2019, realizados com seres humanos, que possuíam no título, resumo ou corpo do texto alguma relação com o objetivo da pesquisa. Foram excluídos os artigos que não possuíam o resumo ou o texto completo nas bases consultadas, artigos repetidos, revisão, outras infecções pulmonares e também artigos que não continham teste de função pulmonar pela espirometria e aqueles que não avaliaram crianças com histórico de BVA antes dos 2 anos de idade. As buscas foram limitadas a estudos publicados nos últimos dez anos com o intuito de enfocar o recente interesse e as evidências científicas nessa área. O processo de seleção dos artigos está descrito em fluxograma apresentado na Figura 1.

A partir da seleção dos resumos dos estudos encontrados, foram recuperados os artigos com texto completo. Após a leitura dos artigos na íntegra, foram extraídos os seguintes dados e realizada comparação entre eles: nome dos autores, ano de publicação, país onde a pesquisa foi desenvolvida, desenho do estudo, idade da infecção, principal agente etiológico, idade no teste de função pulmonar, quantidade de crianças que realizaram a espirometria (em ambos grupos), resultados do teste de função pulmonar.

\section{RESULTADOS}

Foram incluídos cinco artigos na presente revisão, cujas características são apresentadas na Tabela 1. Os estudos

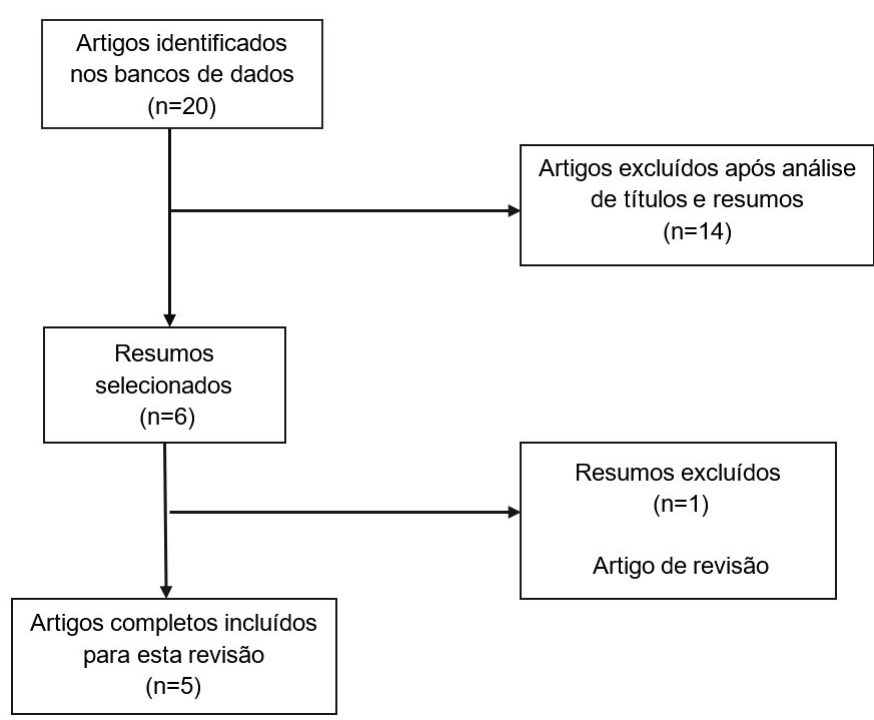

Figura 1. Fluxograma da seleção dos artigos analisados nesta revisão. 


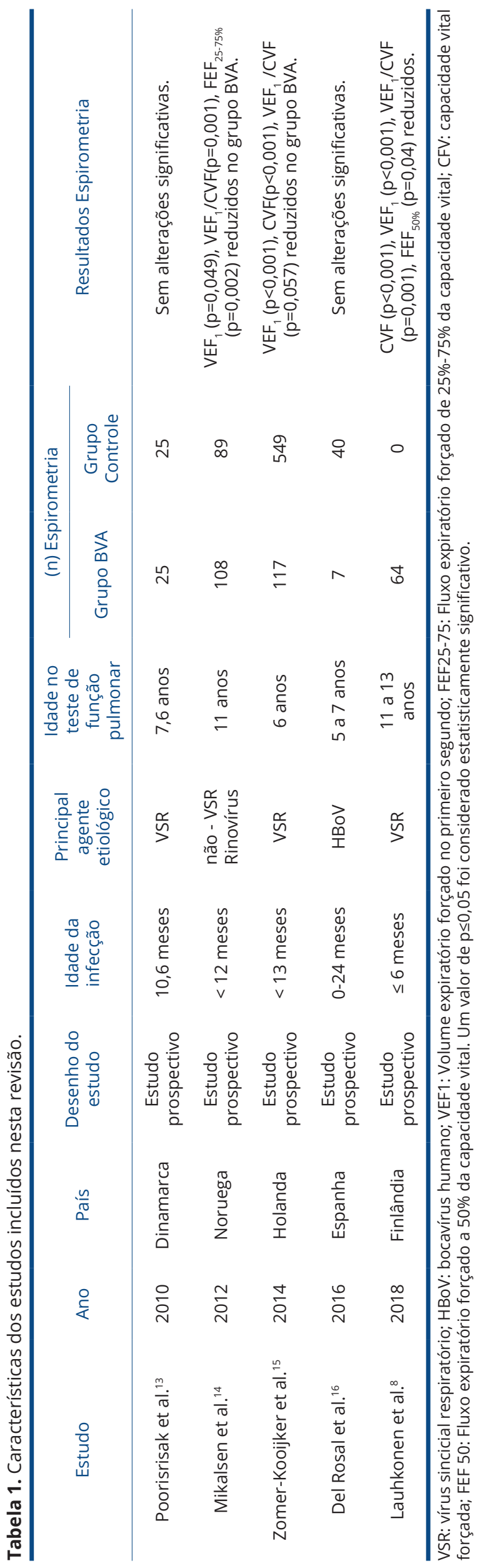


analisados foram realizados nos últimos 10 anos, sendo todos publicados em língua inglesa, em diferentes países (Dinamarca, Noruega, Holanda, Espanha e Finlândia). A maioria dos desenhos dos estudos encontrados foram do tipo coorte prospectiva de acompanhamento hospitalar.

Em todos os estudos selecionados a amostra foi composta por crianças que foram hospitalizadas no momento do diagnóstico de BVA. A idade dos participantes quando hospitalizados por BVA variou de 0 a 24 meses, e a idade na realização da espirometria variou de 5 a 13 anos. O tamanho da amostra para espirometria variou conforme características de cada pesquisa.

Dentre os estudos selecionados, em três deles $8,13,15$ o principal agente etiológico para BVA foi o vírus sincicial respiratório, já nos estudos de Del Rosal et al. ${ }^{16}$ e Mikalsen et al. ${ }^{14}$ os principais agentes etiológicos de infecções respiratórias em crianças pequenas foram o bocavírus humano (HBoV) e o rinovírus, respectivamente. A BVA desencadeada por todos estes vírus foi associada a um risco aumentado de sibilância recorrente, asma $a^{8,13-16} \mathrm{e}$ déficit na função pulmonar; este último observado apenas em três estudos 8,14,15

Nos cinco artigos incluídos nesta revisão, a espirometria fluxo-volume foi realizada de acordo com as diretrizes estabelecidas por cada país, utilizando um equipamento específico local. Foram analisados os parâmetros de função pulmonar CVF, VEF1, relação VEF1/CVF, FEF de 25-75\% da CVF e FEF 50\% da CVF ${ }^{16,17}$ Nas pesquisas de Lauhkonen et al. ${ }^{8}$ e Zomer-Kooijker et al. ${ }^{15}$ as medidas foram realizadas na posição sentada, com um clipe no nariz e a expiração forçada foi repetida até que três curvas aceitáveis fossem obtidas. No estudo de Poorisrisak et al. ${ }^{13}$ o teste de função pulmonar foi realizado de acordo com os critérios da American Thoracic Society. Já nos estudos de Mikalsen et al. ${ }^{14}$ e Del Rosal et al. ${ }^{16}$ a espirometria foi realizada de acordo com diretrizes específicas que não foram detalhadas em suas pesquisas.

Os resultados espirométricos foram comparados com os valores previstos pelas equações de referência padrão e expressos como porcentagens do previsto. O VEF1 e CVF $\geq$ $80 \%$ dos valores previstos foram considerados normais ${ }^{8,16}$ Os testes das crianças que apresentaram alguma infecção do trato respiratório nas últimas 2 semanas foram $\operatorname{adiados}^{14,15}$

Dentre os artigos avaliados, três apresentaram valores reduzidos nas crianças que foram hospitalizados por BVA ${ }^{8,14,15}$ indicando um possível comprometimento pulmonar a longo prazo.

Zomer-Kooijker et al. ${ }^{15}$ mostraram que, quando comparada às crianças não hospitalizadas, a função pulmonar foi significativamente comprometida naquelas com história de hospitalização por VSR (grupo BVA), com redução do VEF1 (diferença média (DM) de 26,8\% previsto), da CVF (DM 26,5\% previsto), do VEF $/$ /CVF (DM $22,0 \%$ ) e aumento da resistência do sistema respiratório (DM 15,6 kPa /L/s). Estes resultados corroboram com os achados de Lauhkonen et al. ${ }^{8}$ porém, neste estudo, a resistência pós-broncodilatador mostrou correlação estatisticamente modesta entre o $\mathrm{VEF}_{1}$ e o VEF$/ \mathrm{CVF}$, mas com diminuição da resistência e aumento percentual no $\mathrm{VEF}_{1}$, indicando reversibilidade patológica.

\section{DISCUSSÃO}

A partir da seleção, leitura e análise dos artigos, percebemos que ainda há poucas evidências relacionada à função pulmonar em crianças com diagnóstico de BVA.

Testes de função pulmonar em pré-escolares (4-6 anos) e escolares (7-12 anos) ${ }^{18}$ são ferramentas fundamentais não apenas como parâmetro clínico, mas também como forma de acompanhamento em longo prazo do crescimento e desenvolvimento pulmonar da infância até a vida adulta, monitorando progresso de possíveis doenças pulmonares crônicas ${ }^{19,20}$.

Segundo Paton et al. ${ }^{21}$ e Gidaris et al. ${ }^{22}$ crianças diagnosticadas com BVA - como primeiro episódio de sibilância - poderão evoluir com asma durante o período pré-escolar e escolar, como consequência da infecção viral e possível relação com fatores ambientais e genéticos ${ }^{13,16}$ A doença sibilante está associada à pior função pulmonar não sendo totalmente claro se esse déficit na função ocorre como consequência do chiado ou predispõe a ele ou a ambos $^{23}$

No estudo de Mikalsen et al. ${ }^{14}$ crianças do grupo BVA (infectadas tanto por VSR quanto por Rinovírus) apresentaram valores menores de $\mathrm{VEF}_{1} \%, \mathrm{FEF}_{25-75 \%} \mathrm{e}$ $\mathrm{VEF}_{1} / \mathrm{CVF}$ quando comparados aos controles. No entanto, estes resultados foram significativos apenas para crianças com BVA causada pelo Rinovírus, mostrando um importante comprometimento pulmonar na presença deste vírus. As funções pulmonares também foram prejudicadas nas crianças hospitalizadas com bronquiolite pelo VSR ${ }^{15}$, causando danos as vias aéreas os quais podem comprometer o desenvolvimento normal da arquitetura pulmonar.

Já nos estudos de Del Rosal et al. ${ }^{16}$ e Poorisrisak et al. ${ }^{13}$ não foram encontradas anormalidades da função pulmonar em pacientes com BVA causada por HBoV, tampouco por VSR. Os autores ${ }^{16}$ relatam que isso talvez se deva a poucos casos de BVA causada por HBoV, possíveis casos de co-infecção quando da hospitalização por BVA ou também rastreio insuficiente de amostras para este vírus. Para Poorisrisak et al. ${ }^{13}$ em estudo realizado com gêmeos semelhantes, há um fator ambiental e talvez genético não revelado que interage levando a diferentes respostas à infecção por VSR.

Outros estudos ${ }^{24-26}$ enfatizaram o papel do VSR como importante patógeno para BVA em lactentes. No entanto, a infecção por outros vírus também vem sendo bastante estudada. Em crianças mais velhas e pré-escolares com sibilos, o rinovírus foi o mais frequentemente detectado ${ }^{25}$ sendo este causador de inflamação, dano e remodelaçãó 
das vias aéreas ${ }^{27}$ Outro importante patógeno respiratório é o HBoV que acomete crianças pequenas infectando o trato respiratório inferior na presença de alta carga viral levando a sibilância aguda e demais sintomas respiratórios ${ }^{16,28}$ Tais dados corroboram com os achados dos estudos incluídos nesta revisão, no qual as manifestações de ambos os vírus levam ao desconforto respiratório, sibilância precoce, risco de asma e, consequentemente, possíveis alterações na função pulmonar.

Zomer-Kooijker et al. ${ }^{15}$ evidenciaram risco três vezes maior nas crianças hospitalizadas com bronquiolite pelo VSR em comparação àquelas não hospitalizadas de desenvolverem asma. No entanto, na coorte conduzida por Mikalsen et al. ${ }^{14}$, a hospitalização por BVA não mostrou associação com o desenvolvimento de asma no final da infância. Em outros estudos ${ }^{14,16}$, o risco de asma subsequente a BVA aumentou consideravelmente em crianças infectadas pelo HBoV e Rinovírus, o que pode persistir até a idade adulta.

Entre esses casos não relacionados ao VSR, encontra-se não apenas rinovírus ${ }^{14}$, mas também o metapneumovírus humano e HBoV ${ }^{16}$ É provável que os mecanismos subjacentes à progressão da asma para cada vírus sejam diferentes ${ }^{25}$; e o efeito a longo prazo destes agentes virais sobre os sintomas ainda necessitam de maiores investigações ${ }^{13}$

Na maioria dos estudos da presente revisão, foram aplicados questionários estruturados para obter informações sobre episódios de sibilos, sintomas de asma, uso de broncodilatadores, doenças alérgicas, além de sintomas durante atividade física, durante o sono, fatores demográficos, exposições ambientais e histórico familiar de doenças respiratórias e atópicas. Esses dados, em dois estudos ${ }^{14,16}$, evidencia as chances maiores de reinternações, uso prolongado de broncodilatadores, cansaço e morbidade respiratória nas crianças do grupo BVA. Como a asma é uma doença crônica com episódios recorrentes de sibilância, tosse e dispneia, ocorre aumento do trabalho respiratório e da percepção do esforço, o que pode comprometer a mecânica respiratória, função muscular respiratória e o condicionamento físico ${ }^{29}$ Nessas condições, essas crianças necessitarão de atendimentos multiprofissionais, cuidados individualizados, adoção de medidas de prevenção e controle de infecções respiratórias de forma a reduzir a morbimortalidade causada pela doença ${ }^{24}$

Conhecer a BVA, sua classificação, principais vírus envolvidos e consequências nos lactentes é de suma importância. Esta é uma parcela da população que poderá apresentar comprometimento e declínio da função pulmonar na idade adulta, sendo um forte preditor de doença pulmonar obstrutiva crônica das vias aéreas ${ }^{15,30}$ o que causa impacto negativo na qualidade de vida.

O maior entendimento sobre o tema pode auxiliar na prática clínica quando esses pacientes reinternam e durante acompanhamentos ambulatoriais por meio de intervenções terapêuticas precoces ${ }^{18}$.

Uma das limitações desta revisão foi a escassez de artigos recentes publicados sobre esse assunto, bem como estudos em outros idiomas realizados em uma gama maior de países.

\section{CONCLUSÃO}

Os achados da presente revisão evidenciam que, na maioria dos artigos incluídos, a BVA no primeiro ano de vida foi fator de risco para sibilância, asma e pior função pulmonar em crianças dos 6 aos 13 anos de idade. Ressaltamos que há uma carência de artigos publicados sobre esse assunto, principalmente em âmbito nacional, bem como a caracterização específica da amostra em relação ao grau de comprometimento da BVA, o que pode ser considerado como limitação deste estudo. Pesquisas futuras devem ser realizadas considerando a gravidade da infecção e possíveis aplicabilidades e desfechos clínicos nesta população.

\section{FONTE DE FINANCIAMENTO}

Nada a declarar

\section{CONFLITO DE INTERESSES}

Nada a declarar

\section{REFERÊNCIAS}

1. Macedo SEC, Menezes AMB, Albernaz E, Post P, Knorst M. Fatores de risco para internação por doença respiratória aguda em crianças até um ano de idade. Rev Saude Publica. 2007;41(3):351-8. http://dx.doi.org/10.1590/S003489102007000300005 . PMid:17515987.

2. American Academy of Pediatrics Subcommittee on Diagnosis and Management of Bronchiolitis. Diagnosis and management of bronchiolitis. Pediatrics. 2006;118(4):177493. http://dx.doi.org/10.1542/peds.2006-2223. PMid:17015575.

3. Lee KK, Hegele RG, Manfreda J, Wooldrage K, Becker AB, Ferguson AC, et al. Relationship of early childhood viral exposu-res to respiratory symptoms, onset of possible asthma and atopyin high risk children: the Canadian Asthma Primary Prevention Study. Pediatr Pulmonol. 2007;42(3):290-7. http://dx.doi.org/10.1002/ppul.20578. PMid:17245731.

4. Viswanathan M, King VJ, Bordley C, Honeycutt AA, Wittenborn J, Jackman AM, et al. Management of bronchiolitis in infants and children: summary [Internet]. Rockville (MD): Agency for Healthcare Research and Quality; 2003. [citado em 2020 Mar 26]. Disponível em: https://www.ncbi.nlm.nih.gov/books/ NBK11847/

5. López Guinea A, Casado Flores J, Martín Sobrino MA, Espínola Docio B, de la Calle Cabrera T, Serrano A, et al. Bronquiolitis grave. Epidemiología y evolución de 284 pacientes. An 
Pediatr (Barc). 2007;67(2):116-22. http://dx.doi.org/10.1016/ S1695-4033(07)70571-4. PMid:17692256.

6. Korppi M, Piippo-Savolainen E, Korhonen K, Remes S. Respiratory morbidity 20 years after RSV infection in infancy. Pediatr Pulmonol. 2004;38(2):155-60. http://dx.doi. org/10.1002/ppul.20058. PMid:15211700.

7. Jartti T, Makela MJ, Vanto T, Ruuskanen O. The link between bronchiolitis and asthma. Infect Dis Clin North Am. 2005;19(3):667-89. http://dx.doi.org/10.1016/j. idc.2005.05.010. PMid:16102655.

8. Lauhkonen E, Riikonen R, Törmänen S, Koponen P, Nuolivirta $\mathrm{K}$, Helminen $\mathrm{M}$, et al. Impulse oscillometry at preschool age is a strong predictor of lung function by flow-volume spirometry in adolescence. Pediatr Pulmonol. 2018;53(5):552-8. http:// dx.doi.org/10.1002/ppul.23977. PMid:29484853.

9. Carbonell-Estrany X, Pérez-Yarza EG, García LS, Cabañas JMG, Bória EV, Atienza BB, et al. Long-term burden and respiratory effects of respiratory syncytial virus hospitalization in preterm infants - the SPRING study. PLoS One. 2015;10(5):e0125422. http://dx.doi.org/10.1371/ journal.pone.0125422. PMid:25955487.

10. Bont L, Steijn M, Van Aalderen WM, Kimpen JL. Impact of wheezing after respiratory syncytial virus infection on healthrelated quality of life. Pediatr Infect Dis J. 2004;23(5):414-7. http://dx.doi.org/10.1097/01.inf.0000122604.32137.29. PMid:15131463.

11. Pereira CAC. Diretrizes para Testes de Função Pulmonar. Espirometria - Diretriz 1. J Pneumol. 2002;28(Supl 3):1-82.

12. Costa $D$, Jamani $M$. Bases fundamentais da espirometria. Rev Bras Fisioter. [Internet]. 2001;5(2):95-102. [citado em 2021 Mar 2]. Disponível em: https://pesquisa.bvsalud.org/portal/ resource/pt /lil-315316

13. Poorisrisak P, Halkjaer LB, Thomsen SF, Stensballe LG, Kyvik $\mathrm{KO}$, Skytthe A, et al. Causal direction between respiratory syncytial virus bronchiolitis and asthma studied in monozygotic twins. Chest. 2010;138(2):338-44. http://dx.doi. org/10.1378/chest.10-0365. PMid:20435661.

14. Mikalsen IB, Halvorsen T, Øymar K. The outcome after severe bronchiolitis is related to gender and virus. Pediatr Allergy Immunol. 2012;23(4):391-8. http://dx.doi.org/10.1111/ j.1399-3038.2012.01283.x. PMid:22435682.

15. Zomer-Kooijker K, van der Ent CK, Ermers MJJ, Uiterwaal CSPM, Rovers MM, Bont LJ. Increased risk of wheeze and decreased lung function after respiratory syncytial virus infection. PLoS One. 2014;9(1):e87162. http://dx.doi. org/10.1371/journal.pone.0087162. PMid:24498037.

16. Del Rosal T, García-García ML, Calvo C, Gozalo F, Pozo F, Casas I. Recurrent wheezing and asthma after bocavirus bronchiolitis. Allergol Immunopathol (Madr). 2016;44(5):410-4. http:// dx.doi.org/10.1016/j.aller.2015.07.004. PMid:26657170.

17. Luisi F, Pinto LA, Marostica L, Jones MH, Stein RT, Pitrez PM. Persistent pulmonary function impairment in children and adolescents with asthma. J Bras Pneumol. 2012 Mar-Abr;38(2):158-66. http://dx.doi.org/10.1590/S180637132012000200003 . PMid:22576422.
18. Friedrich FO, Heinzmann-Filho JP, Birck MA, Pinto LA, Vidal $P C V$. Frequência de sucesso de pré-escolares e escolares com e sem sintomas respiratórios nos testes de função pulmonar. Fisioter Pesqui. 2016;23(2):193-200. http://dx.doi. org/10.1590/1809-2950/15015623022016.

19. Stocks J. Clinical implications of pulmonary function testing in preschool children. Paediatr Respir Rev. 2006;7(Suppl 1):S26-9. http://dx.doi.org/10.1016/j.prrv.2006.04.015. PMid:16798586.

20. Fainardi $V$, Lombardi E. Testes de função pulmonar para monitorar doenças respiratórias em crianças pré-escolares. Acta Biomed. 2018;89(2):148-56. http://dx.doi.org/10.23750/ abm.v89i2.7155.

21. Paton J, Bindels P, McMurray A, Biggins J, Nantanda R, Østergaard MS. A young child with a history of wheeze. NPJ Prim Care Respir Med. 2017;27(1):19. http://dx.doi. org/10.1038/s41533-017-0020-3. PMid:28303014.

22. Gidaris D, Urquhart D, Anthracopoulos MB. They said it was bronchiolitis; is it going to turn into asthma doctor? Respirology. 2014;19(8):1158-64. http://dx.doi.org/10.1111/ resp.12371. PMid:25138566.

23. Belgrave DCM, Buchan I, Bishop C, Lowe L, Simpson A, Custovic A. Trajectories of lung function during childhood. Am J Respir Crit Care Med. 2014;189(9):1101-9. http://dx.doi. org/10.1164/rccm.201309-17000C. PMid:24606581.

24. Brandão HV, Vieira GO, Vieira TO, Cruz ÁA, Guimarães AC, Teles $C$, et al. Acute viral bronchiolitis and risk ofasthma in schoolchildren: analysis of a Brazilian newborn cohort. J Pediatr (Rio J). 2017;93(3):223-9. http://dx.doi.org/10.1016/j. jped.2016.08.004. PMid:27665269.

25. Saglani S. Viral infections and the development of asthma in children. Ther Adv Infect Dis. 2013;1(4):139-50. http:// dx.doi.org/10.1177/2049936113497202. PMid:25165549.

26. Sigurs N, Aljassim F, Kjellman B, Robinson PD, Sigurbergsson $\mathrm{F}$, Bjarnason R, et al. Asthma and allergy patterns over 18 years after severe RSV bronchiolitis in the first year of life. Thorax. 2010;65(12):1045-52. http://dx.doi.org/10.1136/ thx.2009.121582. PMid:20581410.

27. Jackson DJ. The role of rhinovirus infections in the development of early childhood asthma. Curr Opin Allergy Clin Immunol. 2010;10(2):133-8. http://dx.doi.org/10.1097/ ACl.0b013e3283352f7c. PMid:19996738.

28. Allander T, Jartti T, Gupta S, Niesters HG, Lehtinen P, Osterback R, et al. Human Bocavirus and Acute Wheezing in Children. Clin Infect Dis. 2007;44(7):904-10. http://dx.doi. org/10.1086/512196. PMid:17342639.

29. Lanza FC, Corso SD. Fisioterapia no paciente com asma: intervenção baseada em evidências. Braz J Allergy Immunol. 2017;1(1):59-64. http://dx.doi.org/10.5935/25265393.20170008.

30. Stern DA, Morgan WJ, Wright AL, Guerra S, Martinez FD. Poor airway function in early infancy and lung function by age 22 years: a non-selective longitudinal cohort study. Lancet. 2007;370(9589):758-64. http://dx.doi.org/10.1016/ S0140-6736(07)61379-8. PMid:17765525. 